\title{
REGION CLASSIFICATION BASED IMAGE DENOISING USING SHEARLET AND WAVELET TRANSFORMS
}

\author{
Preety D. Swami ${ }^{1}$, Alok Jain ${ }^{2}$ and Dhirendra K. Swami ${ }^{3}$ \\ ${ }^{1}$ Department of Electronics \& Instrumentation Engineering, \\ SATI, Vidisha, India \\ preetydswami@yahoo.com \\ ${ }^{2}$ Department of Electronics \& Instrumentation Engineering, \\ SATI, Vidisha, India \\ alokjain6erediffmail.com \\ ${ }^{3}$ Department of Computer Science and Engineering, VNSIT, Bhopal, India \\ dhirendrakswami@gmail.com
}

\begin{abstract}
This paper proposes a neural network based region classification technique that classifies regions in an image into two classes: textures and homogenous regions. The classification is based on training a neural network with statistical parameters belonging to the regions of interest. An application of this classification method is applied in image denoising by applying different transforms to the two different classes. Texture is denoised by shearlets while homogenous regions are denoised by wavelets. The denoised results show better performance than either of the transforms applied independently. The proposed algorithm successfully reduces the mean square error of the denoised result and provides perceptually good results.
\end{abstract}

\section{KEYWORDS}

Classification, Image denoising, Neural Network, Shearlets, Wavelets

\section{INTRODUCTION}

Extraction of texture regions in an image is required for interpretation of data and is a challenging job. Mostly, the methods that are employed to characterize textures are statistical in nature. Some other methods that extract texture features are those that use Gabor filtering, fractal dimensions, and wavelet transform [1]. The importance of texture detection is important with the perspective of image enhancement, image segmentation and content classification [2]. In this work a classification scheme is proposed that classifies regions in an image into homogenous regions and textures. The effectiveness of this scheme is proved by applying different transformations to the two classified areas in the image. Thus, this algorithm can be used to denoise images using a hybrid of transforms which gives better results than when denoising is done using a single transform.

The wavelet transform has proved to be a powerful tool for image denoising in the past two decades. The pioneering work of Donoho et al. [3] for image denoising paved the way for many

David C. Wyld et al. (Eds) : CST, ITCS, JSE, SIP, ARIA, DMS - 2014

pp. 241-248, 2014. (C) CS \& IT-CSCP 2014

DOI : $10.5121 /$ csit.2014.4122 
researchers to further exploit the multiresolution transforms for denoising purpose. The traditional wavelet transform, although, is good at denoising point singularities in signals, fails at the line singularities such as edges and at textures present in an image. This has led to the need of developing new transforms that may overcome the limitations posed by wavelets. The flowering of many multiresolution transforms, such as, brushlets [4], wedgelets [5], ridgelets [6], curvelets [7], bandelets [8], contourlets [9], waveatoms [10], shearlets [11] and ripplets [12] has provided a handful of options for image denoising. However, the selection of a particular transform is a bit difficult, as, each of these transforms perform sparsely in specific areas of an image. A transform providing sparse representation in smooth areas may not provide sparsity at the edges or textures and vice versa.

Researchers are continuously in search of methods and transforms that can denoise the variations in an image with perfect reconstruction. For the past few years, denoising techniques, based on combination of multiple transforms have evolved. In [13], Ma et al. proposed a denoising algorithm to perform pixel fusion to result images of curvelets and wavelets approaches. The noisy image is denoised using curvelets as well as using HMT based wavelets. Then image regions are analysed with quadtree decomposition. Weighted pixel fusion method is employed to obtain the final result image.

The discrete curvelet transform can code image edges more efficiently than the wavelet transform [14-16]. On the other hand, wavelet transform, codes homogenous areas better than curvelet transform. In [14] two combinations of time invariant wavelet and curvelet transforms are used for denoising of SAR images. Both methods use the wavelet transform to denoise homogeneous areas and the curvelet transform to denoise areas with edges. The segmentation between homogeneous areas and areas with edges is done by using total variation segmentation. In [16] the areas containing edges are denoised using spatially adaptive context modelling of curvelet transform coefficients, while the remaining homogenous regions are recovered through spatially adaptive context modelling of wavelet transform coefficients. The areas containing edges and those that do not contain edges are segmented in the space domain by calculating a variance image and then thresholding it. In [17], three combinations of undecimated wavelet and nonsubsampled contourlet transforms are used for denoising of SAR images. Two methods use the wavelet transform to denoise homogeneous areas and the nonsubsampled contourlet transform to denoise areas with edges. The segmentation between homogeneous areas and areas with edges is done by using total variation segmentation. The third method is a linear averaging of the two denoising methods. A thresholding in the wavelet and contourlet domain is done by non-linear functions which are adapted for each selected subband.

Authors in [18] combine wavelet transform with both the ridgelet and the curvelet transform. The residual image gives the information about the efficiency of the method as no features are seen in it. In [19] BayesShrink wavelet is combined with BayesShrink ridgelet denoising method which performs better than each method individually. The proposed combined denoising method gains the advantage of each filter in its specific domain, i.e., wavelet for natural and ridgelet for straight regions, and produces better and smoother results, both visually and in terms of SNR.

The work in [20] utilizes features of wavelet and curvelet transform, separately and adaptively, in different regions of an image, which are identified by variance approach. The homogenous regions are denoised by wavelets and edgy information is obtained with curvelet transform. The spatially adaptive fusion technique fuses the denoised information obtained from the two transforms.

Authors in [21] proposed a multiscale and multidirectional image representation method named CBlet transform. It combines the contourlet transform with the bandeletization procedure. The contourlet transform captures image discontinuous points and links them into linear structures. 
These linear structures are analysed adaptively by the bandeletization procedure and removes their correlation. The results of the fusion of denoised data from brushlet and wavelet thresholding methods are presented in [22]. Texture-based brushlet denoising is well suited for enhancement of physiological information while wavelet-based denoising is better suited for enhancement of anatomical contours. A three-dimensional multiscale edge-based data fusion algorithm is applied to combine enhanced data from these two independent denoising methods. In [23] a method is proposed for denoising in which firstly, the DTCWT is employed to obtain subbands, and then bandeletization is implemented in each subband. At last, Bayes soft-threshold shrinkage denoising in bandelet transform domain is implemented. Image with highly directional can be efficiently denoised by this method.

Wavelets and compactly supported shearlets sparsely represent point and curvilinear singularities, respectively. [24] presents an image separation method for separating images into point and curvelike parts by employing a combined dictionary consisting of wavelets and shearlets. In this, it is assumed that noise cannot be represented sparsely by either one of the two representation systems. Thus, noise can be captured in the residual.

In one of our previous works [25], an image denoising method which adaptively combines the features of wavelets, wave atoms and curvelets was proposed. It employs wavelet shrinkage to denoise the smooth regions in the image while wave atoms are employed to denoise the textures and the edges take advantage of curvelet denoising.

This paper proposes a classification technique for segmenting an image into two categories: homogenous regions and texture. The proposed classification algorithm is based on training a neural network using samples from images and some statistical measures pertaining to the two above mentioned categories present in a natural image. The neural network classifier accurately assigns the classes to the different regions. Once the classification is done the results can be used to denoise a noisy image. This work employs a combination of wavelets and shearlets for the purpose of denoising.

The organization of the paper is as follows. Section 2 deals with the proposed region classification algorithm using neural network, along with, an application of the algorithm for image denoising. Experimental results are analysed in Section 3. Finally conclusion is given in Section 4.

\section{PROPOSED METHOD}

In image denoising, identification of smooth, texture and other regions is a frequent requirement. Various methods have been proposed in the literature for separating smooth, texture and other regions present in the image. Texture is different from smooth areas in that they have some randomness in location, size and orientation of the texture elements [2]. Several texture descriptors are available for identifying textures. These include Gray Level Co-occurrence Matrix (GLCM), contrast, directionality, placement rules, Markov Random Field models, and filtering in the transform domain [1]. This task of classification can also be attained by machine learning in a supervised manner. In this section an attempt has been made to use neural network for texture and smooth region identification.

In this proposed classification method, training of a neural network is required which classifies an incoming data (image block) to either smooth class or texture class. For this task, the neural network is trained on some blocks of texture patches and some blocks of smooth region patches. These patches can be extracted from the image of Barbara as shown in Figure 1. In this figure, the region inside the red rectangles, are used to train the texture class and the areas inside the blue 
squares are used to provide training of smooth regions. Each patch is divided into blocks of size $9 \times 9$, and these 81 pixel intensity samples are provided as inputs to the neural network.

Along with the 81 pixel intensity values of an image block (texture or smooth), three texture descriptors are also used to train the neural network. These descriptors are variance, contrast and connected component count. Each descriptor is calculated for a window size of $9 \times 9$.

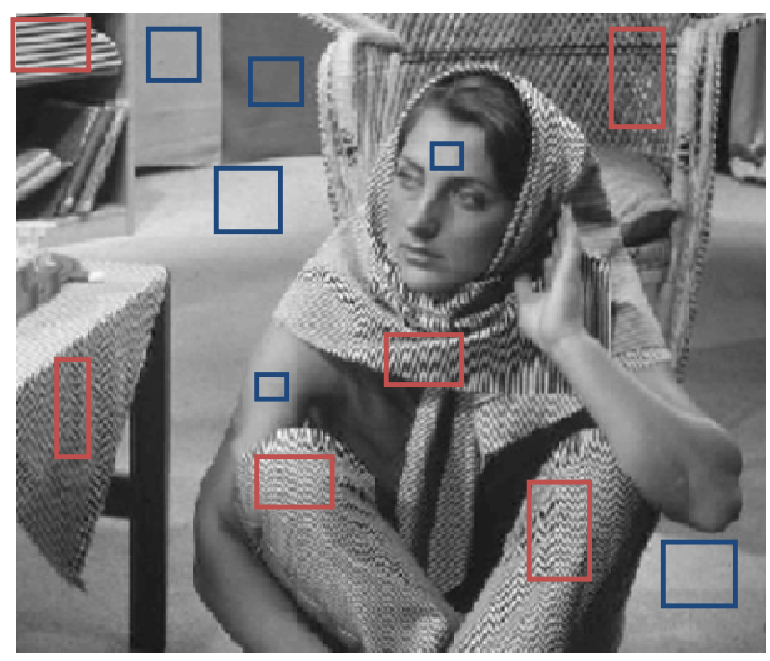

Figure 1. Sample extraction for neural network training. Pixels inside the red rectangle train the texture class and pixels inside the blue square train the class corresponding to smooth regions.

The variance inside a window is calculated as the square of the standard deviation. For the calculation of connected components the pixels inside the $9 \times 9$ window are divided into two groups. For this the average grey level is computed and the pixel intensity greater than the average are assigned to the first group and the remaining pixels belong to the second group. A binary image of the $9 \times 9$ block is created in which the group one pixels are set to zero and group two pixels are set to 1 . Finally connected components are computed by using the Matlab command 'bwlabel'. Contrast can be calculated by calculating the difference between the averages of the above discussed two groups of pixels. All the three descriptors are calculated for the Barbara image and are depicted in Figure 2.

The use of classification by the proposed method is applied for denoising of Barbara image. After classification the image regions are classified into two regions, smooth and texture. These regions will be denoised by two different transforms according to the sparse behaviour offered by them in the respective regions. Wavelets efficiently denoise the smooth regions but fail at the edges and in texture regions as they are less sparse in these regions. Shearlets are sparser than wavelets for reconstructing edges and texture but, at the same time, introduce artifacts in the smooth regions. Thus in this work wavelets denoise the smooth regions and shearlets denoise the textures. The noisy image is denoised first by wavelets [26] and then by shearlets [11]. The proposed classification scheme is applied to the wavelet denoised image for region classification and its result is used to fuse the two denoised images. The regions belonging to smooth areas are replaced by the respective wavelet denoised pixels and the regions belonging to texture are replaced by the corresponding shearlet denoised pixels. Thus both transforms work individually and effectively in different regions of the image. 


\section{EXPERIMENTS AND RESULTS}

Feed Forward neural network is commonly used for classification task using back propagation algorithm. In our experiments, a feed forward neural network has been trained to identify a small image window for its smooth or textured nature. For this purpose experiments have been conducted on the popularly used image of Barbara. A set of $9 \times 9$ pixel subsets of Barbara have been taken and have been labelled as smooth or texture by human observation. Sufficiently large set of such training samples have been set up to achieve supervised classification using

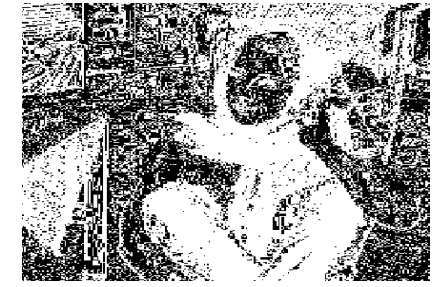

(a)

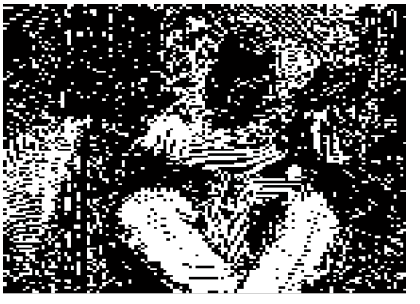

(b)

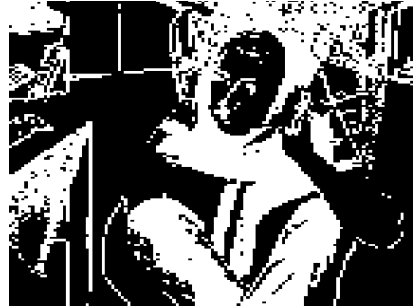

(c)

Figure 2. Texture descriptors for Barbara (a) Contrast, (b) Component Count and (c) Variance.

neural network. In addition to the image intensity pixel values of training data's $9 \times 9$ image window subsets, features like variance, contrast and connected component count have also been extracted for training data sample to increase distinguishing ability of different regions. Algorithms employed for extraction of texture descriptors used in this work are explained in detail in [2].

Topology of the network used is explained below:

- Intensity of the image pixel values and the texture descriptors features of the training sample $(9 \times 9$ block) form the input vector to the neural network. For these training samples the target output is known in the form of ' 0 ' for smooth regions and as ' 1 ' for texture regions.

- $\quad$ Number of layers $=2$

Number of neurons in the input layer $=84$. Out of these, 81 neurons correspond to the normalized pixel intensity values of the $9 \times 9$ window. Remaining 3 neurons correspond to the three texture descriptor features: variance, contrast and connected component count.

- Output layer contains 1 node that outputs a ' 0 ' for the class smooth regions or a ' 1 ' for the class texture regions.

- $\quad$ Hidden layer nodes $=6$.

This experiment quite satisfactorily trained the various $9 \times 9$ sub images of Barbara. This was tested by simulating the neural network on the training data, which gave the same desired output. Experiments were conducted in Matlab 7.0. The transfer function of all neurons was chosen as 'tansig'. The backpropagation network training function chosen was 'trainlm' and the backpropagation weight/bias learning function used was 'learngdm'. Testing was done by varying the number of hidden layer nodes from 3 to 60 . Best results were obtained while taking 6 neurons in the hidden layer. In this training network, with 6 nodes in the hidden layer, the mean squared error (MSE) was of the order of $10^{-9}$. 


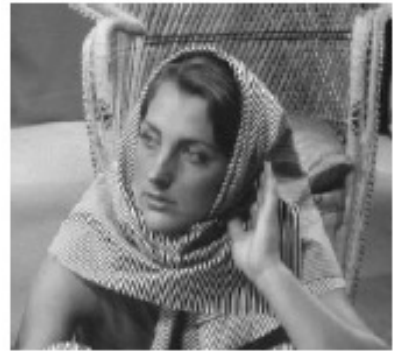

(a)

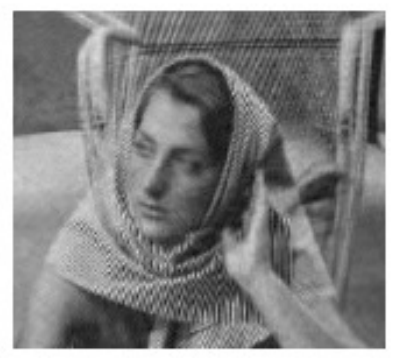

(d)

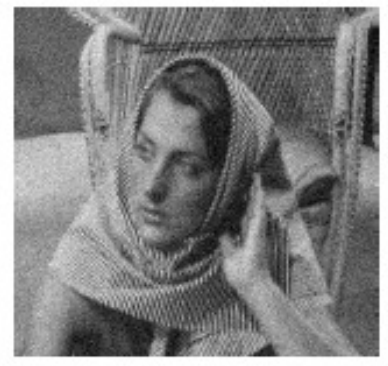

(b)

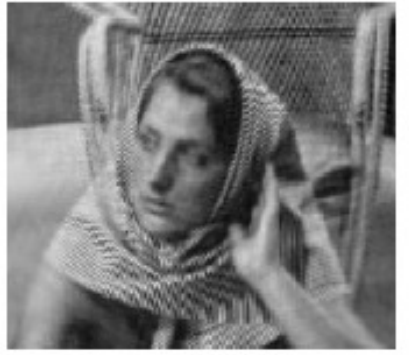

(e)

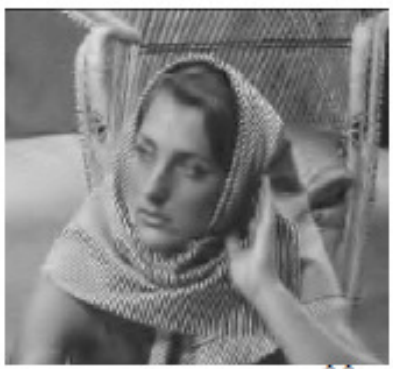

(c)

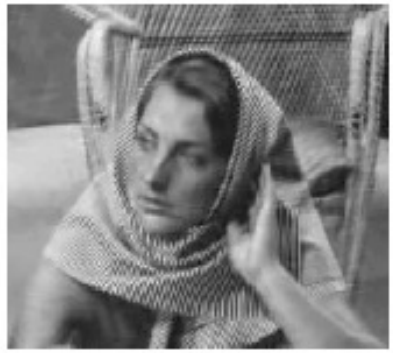

(f)

Figure 3. Denoising resuls of Barbara by different methods. (a) Original (b) Noisy Barbara with noise standard deviation 20 (c) Wavelets [26], PSNR= 29.53dB (d) Shearlets [11], PSNR=28.54dB (e) Wave atoms [10], PSNR=29.31 dB (f) Proposed method, PSNR= 29.83dB

To compare the application of the proposed region classification method in image denoising, a $512 \times 512$ sized white Gaussian noise is added to the Barbara image of same size. The software for shearlets has been downloaded from [27]. The parameter employed for comparison of the denoised results is the Peak Signal to Noise Ratio (PSNR). In Figure 3, the proposed results are compared with the denoising results employing individually the wavelets, the shearlets as well as the waveatoms, which is considered for its high efficiency in denoising textures. The software for waveatoms has been downloaded from [28]. It can be observed from the figure that the proposed method yields the best PSNR. The element like artifacts present in shearlet and waveatom denoising are not present in the proposed denoising method and at the same time textures are visible with clarity.

\section{CONCLUSIONS}

In this paper, a classification method to separate texture from smooth regions of an image is proposed. For classification into the two above mentioned regions, a neural network is trained with sample parameters taken from smooth and texture images. The parameters selected to train the network are the pixel intensities inside a small window and variance, contrast and the connected component count of the same window. Testing of the network provides successful separation of the smooth and the textured regions.

The effectiveness of the algorithm is tested by applying a wavelet-shearlet combination for denoising of natural and texture rich Barbara image. The proposed denoised results are compared with the results of denoising the image individually by wavelets, shearlets and wave atoms. It is observed that using the proposed classification technique in denoising of images, improves the 
PSNR significantly and results in a perceptually cleaner image as compared to employing any of these transforms in individuality.

Future research includes selection of a vivid variety of samples of each category from a large group of natural images for training of the network. The training function and training parameters of the network can also be changed and tested. The effect of a change in window size for more efficient texture descriptors can also be observed.

\section{REFERENCES}

[1] L. Semler \& L. Dettori, (2006) "Curvelet-based texture classification of tissues in computed tomography”, IEEE International Conference on Image Processing (ICIP06), Atlanta, GA, USA, 8-11 Oct., pp 2165-2168.

[2] R. Bergman, H. Nachlieli, \& G. Ruckenstein, (2008) "Detection of textured areas in natural images using an indicator based on component counts", J. Electronic Imaging, Vol. 17, No. 4, pp 043003.

[3] D. L. Donoho \& I. M. Johnstone, (1994) "Ideal spatial adaptation by wavelet shrinkage," Biometrika”, 81, 3, pp 425-455.

[4] F. G. Meyer \& R. R. Coifman, (1997) "Brushlets: A tool for directional image analysis and image compression", Applied and Computational Harmonic Analysis, Vol. 4, pp 147-187.

[5] D. L. Donoho, (1999) "Wedgelets: Nearly minimax estimation of edges", The Annals of Statistics, Vol. 27, No. 3, pp 859-897.

[6] E. J. Candes \& D. L. Donoho, (1999) "Ridgelets: a key to higher-dimensional intermittency?", hil. Trans. R. Soc. London. A., Vol. 357, No. 1760, pp 2495-2509.

[7] J. L. Starck, E. J. Candes \& D. L. Donoho, (2002) "The curvelet transform for image denoising", IEEE Trans. Image Process., Vol. 11, No. 6, pp 670-684.

[8] E. L. Pennec \& S. Mallat, (2005) "Sparse geometric image representations with bandelets", IEEE Trans. Image Process., Vol. 14, No. 4, pp 423-438.

[9] M. N. Do \& M. Vetterli, (2005) "The contourlet transform: an efficient directional multiresolution image representation", IEEE Transactions on Image Process., Vol. 14, No. 12, pp 2091-2106.

[10] L. Demanet \& L. Ying, (2007) "Wave atoms and sparsity of oscillatory patterns", Applied and Computational Harmonic Analysis, Vol. 23, No. 3, pp 368-387.

[11] K. Guo \& D. Labate, (2007) "Optimally sparse multidimensional representation using shearlets", SIAM J. Math Anal., Vol. 39, pp 298-318.

[12] J. Xu, L. Yang \& D. Wu, (2010) "Ripplet: A new transform for image processing”, Journal of Vis. Commun. Image R., Vol. 21, pp 627-639.

[13] L. Ma, J. Ma \& Y. Shen, (2007) "Pixel fusion based curvelets and wavelets denoise algorithm", Engineering Letters, Vol. 14, No. 2, pp 130-134.

[14] J. R. Sveinsson \& J. A. Benediktsson, (2007) "Combined wavelet and curvelet denoising of SAR images using TV segmentation", Proc. of IEEE International Geoscience and Remote Sensing Symposium, pp 503-506.

[15] Y. Li, S. Zhang \& J. Hu, (2010) "Combining curvelet transform and wavelet transform for image denoising", Proc. of 6th International Conference on Intelligent Computing, pp 317-324.

[16] P. D. Swami \& A. Jain, (2012) "Segmentation based combined wavelet-curvelet approach for image denoising”, International Journal of Information Engineering, Vol.2, No. 1, pp 32-37.

[17] J. R. Sveinsson \& J. A. Benediktsson, (2008) "Combined wavelet and contourlet denoising of SAR images", Proc. of IEEE International Geoscience and Remote Sensing Symposium," pp 1150-1153.

[18] J. L. Starck, D. L. Donoho \& E. Candes, (2001) "Very high quality image restoration by combining wavelets and curvelets", Proc. of SPIE conference on Signal and Image Processing: Wavelets: Applications in Signal and Image Processing IX, Vol. 4478, pp 9-19.

[19] N. N. Kachouie \& P. Fieguth, (2006) "A combined Bayesshrink wavelet-ridgelet technique for image denoising", Proc. of IEEE International Conference on Multimedia and Expo, Toronto, Ont., pp 19171920, 9-12 July.

[20] G. G. Bhutada, R. S. Anand \& S. C. Saxena, (2011) "Edge preserved image enhancement using adaptive fusion of images denoised by wavelet and curvelet transform", Digital Signal Processing, Vol. 21, pp 118-130. 
[21] B. Song, L. Xu \& W. Sun, (2007) "Image denoising using hybrid contourlet and bandelet transforms", IEEE Proc. of Fourth International Conference on Image and Graphics, Chengdu, Sichuan, China, pp 71-74.

[22] A. Laine, E. D. Angelini, Y. Jin, P. D. Esser \& R. V. Heertum, (2004) "Fusion of brushlet and wavelet denoising methods for nuclear images", International Symposium on Biomedical Imaging (ISBI), Arlington, VA, USA, pp 1187-1191.

[23] Z. Song \& L. Yuanpeng, (2009) "A novel image denosing scheme via combining dual-tree complex wavelet transform and bandelets", Proc. of Third International Symposium on Intelligent Information Technology Application, Nanchang, China, Vol. 1, pp 509-512.

[24] G. Kutyniok \& W.Q Lim, (2012) "Image separation using wavelets and shearlets", curves and surfaces, Lecture Notes in Computer Science, Springer, Vol. 6920, pp 416-430.

[25] P. D. Swami \& A. Jain, (2012) "Image denoising by adaptive fusion of decomposed images restored using wave atom, curvelet and wavelet transform", Published online in Springer Journal of Signal, Image and Video Processing, DOI: 10.1007/s11760-012-0343-z.

[26] A. Pizurica \& W. Philips, (2006) "Estimating the probability of the presence of a signal of interest in multiresolution single- and multiband image denoising", IEEE Trans. Image Process., Vol. 15, No. 3, pp 654-665.

[27] www.shearlab.org

[28] www.waveatom.org

\section{AUTHORS}

Preety D Swami received the B.E. degree in Electronics and Instrumentation from Samrat Ashok Technological Institute, Vidisha, in 1992, the M.Tech. degree in Digital Communication from Maulana Azad National Institute of Technology, Bhopal, in 2008 and the $\mathrm{Ph}$. D. degree in Electronics engineering from RGPV, Bhopal in 2013. She has 15 research publications in journals and conference proceedings. She has received two best paper awards in conferences. She is a member of IET and member for life, ISTE. Her research interests include transforms, signal processing and image processing.

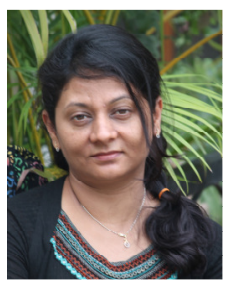

Alok Jain was born in Vidisha, India in 1966. He received his B.E. (Electronics \& Instrumentation) degree from Samrat Ashok Technological Institute, Vidisha, M.Tech. (Computer Science and Technology) from IIT Roorkee (University of Roorkee), in 1988 and 1992, respectively. He obtained his Ph.D. degree from Thapar University (erstwhile Thapar Institute of Engineering and Technology), Patiala, India, in 2006. He is presently serving as a Professor and Head in the Department of Electronics \& Instrumentation Engineering, Samrat Ashok Technological Institute, Vidisha, India. He has published more than 50 papers in journals and conference proceedings of international repute.

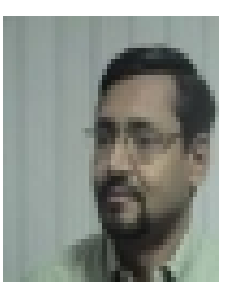

He authored two monographs related to filterbank and Transmultiplexer and two books on power electronics. He co-chaired the session in Int. Conf. SCI 2004 held at Orlando, USA. Dr. Jain is a life member of IE(I), IETE, ISTE, BMESI, Instrument Society of India and is the member of IET. His current research interests include digital signal processing, multirate signal processing, filterbanks, and their applications in image processing.

Dhirendra K Swami is presently Group Director, VNS Group of Institutions, Bhopal. Before joining VNS in 2008, he has served Samrat Ashok Technological Institute (SATI), Vidisha for 20 years. He obtained his $\mathrm{Ph}$. D. in Computer Science and Engineering in 2007 from Rajiv Gandhi Technological University, Bhopal. He completed M. Tech in Computer Applications from IIT, Delhi in 1994 and he completed M.Sc. in Applied Mathematics in 1988. During 25 years of teaching, he has taught various courses in computer science to the students of BE, M. Tech. and MCA students. He has more than 30 research publications in journals and in conference proceedings of repute. He has edited one book and authored a book on Basic Computer Engineering. He is a member for life ISTE and senior life member of CSI. Areas of his special interest include Data Mining, DBMS, Object Oriented System and software Engineering. 